\title{
PERSISTENCE OF THE NORMALIZED EIGENVECTORS OF A PERTURBED OPERATOR IN THE VARIATIONAL CASE
}

\author{
RAFFAELE CHIAPPINELLI \\ Dipartimento di Scienze Matematiche ed Informatiche, Pian dei Mantellini 44, I-53100 Siena, Italy \\ e-mail: chiappinelli@unisi.it \\ and MASSIMO FURI and MARIA PATRIZIA PERA \\ Dipartimento di Matematica Applicata 'G. Sansone', Via S. Marta 3, I-50139 Florence, Italy \\ e-mails:massimo.furi@unifi.it,mpatrizia.pera@unifi.it
}

(Received 20 April 2012; accepted 14 October 2012; first published online 25 February 2013)

\begin{abstract}
Let $H$ be a real Hilbert space and denote by $S$ its unit sphere. Consider the nonlinear eigenvalue problem $A x+\varepsilon B(x)=\delta x$, where $A: H \rightarrow H$ is a bounded self-adjoint (linear) operator with nontrivial kernel $\operatorname{Ker} A$, and $B: H \rightarrow H$ is a (possibly) nonlinear perturbation term. A unit eigenvector $x_{0} \in S \cap \operatorname{Ker} A$ of $A$ (thus corresponding to the eigenvalue $\delta=0$, which we assume to be isolated) is said to be persistent, or a bifurcation point (from the sphere $S \cap \operatorname{Ker} A$ ), if it is close to solutions $x \in S$ of the above equation for small values of the parameters $\delta \in \mathbb{R}$ and $\varepsilon \neq 0$. In this paper, we prove that if $B$ is a $C^{1}$ gradient mapping and the eigenvalue $\delta=0$ has finite multiplicity, then the sphere $S \cap \operatorname{Ker} A$ contains at least one bifurcation point, and at least two provided that a supplementary condition on the potential of $B$ is satisfied. These results add to those already proved in the non-variational case, where the multiplicity of the eigenvalue is required to be odd.
\end{abstract}

2000 Mathematics Subject Classification. Primary 47A55, Secondary 47J05, 47J10, $47 \mathrm{~J} 15$.

1. Introduction and statement of the results. Let $S$ be the unit sphere in a real Hilbert space $H$ and consider the equation

$$
T x+\varepsilon B(x)=\lambda x
$$

in the unknown $(\lambda, \varepsilon, x) \in \mathbb{R} \times \mathbb{R} \times S$, where $T$ is a bounded self-adjoint (linear) operator in $H$ and $B$ is a (possibly nonlinear) continuous operator in $H$. We consider the following bifurcation problem for (1.1): suppose that $\lambda_{0}$ is an eigenvalue of $T$, let $\operatorname{Ker}\left(T-\lambda_{0} I\right)$ be the corresponding eigenspace, and put

$$
S_{0}:=S \cap \operatorname{Ker}\left(T-\lambda_{0} I\right)
$$

Then, (1.1) possesses the family

$$
Z_{0}:=\left\{\left(\lambda_{0}, 0, v\right) \mid v \in S_{0}\right\}
$$

of trivial solutions. A trivial solution $\left(\lambda_{0}, 0, v_{0}\right) \in Z_{0}$ - essentially, a normalized $\lambda_{0}$-eigenvector $v_{0}$ of $T$ - is said to be a bifurcation point from $Z_{0}$ for the solutions of (1.1), or briefly a bifurcation point from $Z_{0}$ for (1.1), if any neighbourhood of $\left(\lambda_{0}, 0, v_{0}\right)$ 
in $\mathbb{R} \times \mathbb{R} \times S$ contains nontrivial solutions - that is, solutions not in $Z_{0}$ - of (1.1). If we assume that $\lambda_{0}$ is an isolated eigenvalue, then any nontrivial solution $(\lambda, \varepsilon, x)$ of (1.1) with $\lambda$ close to $\lambda_{0}$ must have $\varepsilon \neq 0$. Therefore, $v_{0} \in S_{0}$ is a bifurcation point in the above sense if and only if there exists a sequence $\left\{\left(\lambda_{n}, \varepsilon_{n}, x_{n}\right)\right\}$ in $\mathbb{R} \times \mathbb{R} \backslash\{0\} \times S$ which converges to $\left(\lambda_{0}, 0, v_{0}\right)$ and such that $T x_{n}+\varepsilon_{n} B\left(x_{n}\right)=\lambda_{n} x_{n}, \forall n \in n$. In this case, we say equivalently that $v_{0}$ is a persistent normalized $\lambda_{0}$-eigenvector of $T$.

In the papers [2-4], we have discussed both necessary conditions and sufficient conditions for this particular kind of bifurcation to occur when $\lambda_{0}$ is an isolated eigenvalue of $T$ of finite multiplicity (algebraic and geometric multiplicity coincide for self-adjoint operators). Particularly in [3], using as a main tool the Lefschetz fixed-point theorem (see [9] for example) we have shown that bifurcation from $Z_{0}$ does indeed take place at some point of $Z_{0}$ provided that $B$ is of class $C^{1}$ and the multiplicity of $\lambda_{0}$ is $o d d$. It is well known that a similar result holds for bifurcation in the classical sense, that is for the one-parameter equation

$$
T x+B(x)=\lambda x,
$$

where $B$ is $C^{1}$ near $x=0$ and satisfies the conditions $B(0)=B^{\prime}(0)=0$. Here, the trivial solutions are $\{(\lambda, 0) \mid \lambda \in \mathbb{R}\}$, and so one is interested in bifurcation from this line at a given point $\left(\lambda_{0}, 0\right)$. It is equally well known that if in addition $B$ is a gradient operator so that (1.2) has a variational character - then the requirement of oddness about the multiplicity of $\lambda_{0}$ can be removed. For the precise statement of these basic results about (1.2), we refer for instance to $[\mathbf{1}, \mathbf{5}, \mathbf{6}, \mathbf{1 0}, \mathbf{1 2}, \mathbf{1 3}]$.

In this paper, we give more evidence of the similarity existing between (1.1) and (1.2), as we show that when $B$ is a gradient, there is bifurcation from $Z_{0}$ irrespective of the (finite) multiplicity of $\lambda_{0}$. The formal statement is given in Theorem 1.1 below. Before doing this, we wish to remark that 'nonclassical' bifurcation problems of the kind considered here have been proposed by several authors: see in particular Ambrosetti and Prodi [11], who called them atypical bifurcation problems and Furi and Pera [8], who introduced the related concept of cobifurcation; see in addition the paper [7], where a unifying framework for both classical and 'atypical' bifurcation is proposed. Also, we believe that the analysis of (1.1) is far from complete and in fact many open problems await attention, for instance the possible extension of the results in [3] to Banach space operators.

It is now time to state formally the main result of the present paper, and before this we make precise our assumptions about the nonlinear term $B$. These are as follows:

(B1) $B: U \rightarrow H$ is a $C^{1}$ operator defined on a neighbourhood $U$ of $S$;

(BG) $B$ is the gradient of a functional: that is, there exists a differentiable functional $b$ defined on $U$ such that

$$
\langle B(x), y\rangle=b^{\prime}(x) y, \quad \forall x \in U, \forall y \in H .
$$

In (1.3), $\langle\cdot, \cdot\rangle$ denotes the scalar product in $H$ and $b^{\prime}(x)$ denotes the (Fréchet) derivative of $b$ at the point $x \in U$. We then write for short $B=\nabla b$.

THEOREM 1.1. Let $T$ be a bounded self-adjoint operator in a real Hilbert space $H$, and let $\lambda_{0}$ be an isolated eigenvalue of $T$ of finite multiplicity. If $B$ satisfies (B1) and $(B G)$, then there exists at least one bifurcation point from $Z_{0}$ for (1.1).

For the proof of Theorem 1.1, we take advantage of a technical lemma proved in [3] and of an idea of Stuart [13]. The former is needed for an appropriate global 
reduction of (1.1) to a finite-dimensional equation in $\operatorname{Ker}\left(T-\lambda_{0} I\right)$, while the latter permits to identify the solutions of this equation - for $\varepsilon$ fixed - as the critical points of a $C^{1}$ functional $\alpha_{\varepsilon}$ (related to the quadratic form of $T$ ) on a compact $C^{1}$ manifold $M_{\varepsilon}$ which is essentially a deformation of $S_{0}$. Then the minimum and the maximum points of $\alpha_{\varepsilon}$ on $M_{\varepsilon}$ give approximate solutions $v_{\varepsilon}$, and letting $\varepsilon \rightarrow 0$ in a suitable manner yields the desired bifurcation point in $S_{0}$.

This passage to the limit can be better controlled, and permits a considerable improvement of Theorem 1.1, when $B$ is bounded in $U$ (or in a smaller neighbourhood of $S$ ). Indeed in this case we show - supposing in addition that $b$ is not constant on $S_{0}$ - that there are (at least) two distinct bifurcation points for (1.1), which moreover have the property of minimizing (resp. maximizing) $b$ over $S_{0}$. This is formally stated as Theorem 2.1 in the next section. It is not clear to us whether the condition that $b$ is not constant on $S_{0}$ is essential (so that this adds to the open problems about (1.1)). For further remarks about (1.1) we refer to the review [4], where the results of the present paper were announced.

\section{Proof of the results. Set}

$$
A=T-\lambda_{0} I, \quad \delta=\lambda-\lambda_{0}
$$

and write (1.1) as

$$
A x+\varepsilon B(x)=\delta x .
$$

Also put

$$
N=\operatorname{Ker} A=\{x \in H: A x=0\}, \quad W=\operatorname{Im} A=\{A x: x \in H\} .
$$

Then, by our assumptions on $T$ and $\lambda_{0}, H$ is the orthogonal sum

$$
H=N \oplus W .
$$

Let $P$ and $Q=I-P$ be the orthogonal projections onto $N$ and $W$ respectively; then writing $x=P x+Q x=: v+w$ according to (2.2) and applying in turn $P, Q$ to both members of (2.1), we see that this equation is equivalent to the system

$$
\begin{gathered}
\varepsilon P B(v+w)=\delta v, \\
A w+\varepsilon Q B(v+w)=\delta w .
\end{gathered}
$$

In the first part of our analysis we proceed essentially as in [3]. Let $V^{\prime}$ and $W^{\prime}$ be neighbourhoods of $S_{0}$ in $N$ and of 0 in $W$ respectively such that $V^{\prime}+W^{\prime} \subset U$. Identify $H$ with $N \times W$ and consider the map $f$ of $\mathbb{R} \times \mathbb{R} \times V^{\prime} \times W^{\prime}$ into $W$ defined putting

$$
f(\delta, \varepsilon, v, w):=A w+\varepsilon Q B(v+w)-\delta w .
$$

We have

$$
\frac{\partial f}{\partial w}\left(0,0, v_{0}, 0\right)=\left.A\right|_{W}
$$


for any $v_{0} \in V^{\prime}$, and $\left.A\right|_{W}: W \rightarrow W$ is an isomorphism. Then (B1) and a straightforward application of the implicit function theorem guarantee that, given any $v_{0} \in S_{0} \subset V^{\prime}$, equation (2.4) - the so-called complementary equation - can be solved uniquely w.r.t. $w$ for each given $(\delta, \varepsilon, v)$ in a neighbourhood $Y_{0} \subset \mathbb{R} \times \mathbb{R} \times N$ of $\left(0,0, v_{0}\right)$. Moreover if $w(\delta, \varepsilon, v)$ denotes the solution corresponding to $(\delta, \varepsilon, v) \in Y_{0}$, then $w(0,0, v)=0$ for any $v$ and the mapping $(\delta, \varepsilon, v) \rightarrow w(\delta, \varepsilon, v)$ of $Y_{0}$ into $W$ is of class $C^{1}$. Therefore, by definition

$$
A w(\delta, \varepsilon, v)+\varepsilon Q B(v+w(\delta, \varepsilon, v))=\delta w(\delta, \varepsilon, v)
$$

for any $(\delta, \varepsilon, v) \in Y_{0}$; and - replacing $w(\delta, \varepsilon, v)$ in (2.3) - we see that in order to solve (2.1) it is enough to find $(\delta, \varepsilon, v) \in Y_{0}$ satisfying the finite-dimensional equation (the bifurcation equation)

$$
\varepsilon P B(v+w(\delta, \varepsilon, v))=\delta v
$$

and the additional normalization constraint

$$
v+w(\delta, \varepsilon, v) \in S .
$$

However, a strengthened version of the implicit function theorem is needed here in order to solve the complementary equation globally with respect to $S_{0}$. Thus, for $\eta>0$, consider the compact neighbourhood of $S_{0}$

$$
M=\{v \in N:|\|v\|-1| \leq \eta\}
$$

and take $\eta>0$ so small that $M \subset V^{\prime}$. Then applying Lemma 2.2 of [3] with $f$ as in (2.5), $\Omega=\mathbb{R} \times \mathbb{R} \times V^{\prime} \times W^{\prime}$ and $G=\{0\} \times\{0\} \times M \times\{0\}$, it follows that the function $w=w(\delta, \varepsilon, v)$ can be taken to be defined and of class $C^{1}$ on an open neighbourhood $Y_{1}=I_{1} \times J_{1} \times V_{1} \subset \mathbb{R} \times \mathbb{R} \times N$ of $\{0\} \times\{0\} \times M$.

A further reduction can be made on 'eliminating $\delta$ ' from our equations. Indeed, take the scalar product in (2.7) to get

$$
\langle\varepsilon P B(v+w(\delta, \varepsilon, v)), v\rangle=\delta\|v\|^{2} .
$$

Dividing both members of (2.10) by $\|v\|^{2}$ and applying again Lemma 2.2 of [3] to the resulting equation, we see that $\delta$ can be written as a $C^{1}$ function $\delta(\varepsilon, v)$ of $(\varepsilon, v)$, defined on the closure of an open subset $Z=J \times V$ of $\mathbb{R} \times N$ containing $\{0\} \times M$ and such that $\delta(0, v)=0$ for any $v \in V$, and $(\delta(\varepsilon, v), \varepsilon, v) \in Y_{1}$ for $(\varepsilon, v) \in Z$. We shall also assume that the closure $\bar{V}$ of $V$ is compact and does not contain the origin of $N$. For convenience put

$$
\phi(\varepsilon, v):=w(\delta(\varepsilon, v), \varepsilon, v), \quad(\varepsilon, v) \in Z
$$

Then, by definition

$$
\langle\varepsilon P B(v+\phi(\varepsilon, v)), v\rangle=\delta(\varepsilon, v)\|v\|^{2}
$$

for $(\varepsilon, v) \in Z$, and we also have in particular from (2.6):

$$
A \phi(\varepsilon, v)+\varepsilon Q B(v+\phi(\varepsilon, v))=\delta(\varepsilon, v) \phi(\varepsilon, v) .
$$


Moreover, $\phi(0, v)=0$ for $v \in V$ and we see - from (2.7) and (2.8) - that in order to have a nontrivial solution, we need to find $(\varepsilon, v) \in Z, \varepsilon \neq 0$, such that

$$
\varepsilon P B(v+\phi(\varepsilon, v))=\delta(\varepsilon, v) v
$$

and

$$
\|v+\phi(\varepsilon, v)\|^{2}=\|v\|^{2}+\|\phi(\varepsilon, v)\|^{2}=1
$$

A normalized solution $(\delta, \varepsilon, x)$ of the original equation (2.1) will then be given by $(\delta(\varepsilon, v), \varepsilon, v+\phi(\varepsilon, v))$.

To proceed to the main step of the proof, we also put for convenience

$$
F_{\varepsilon}(x):=A x+\varepsilon B(x), \quad \delta_{\varepsilon}(v):=\delta(\varepsilon, v), \quad \phi_{\varepsilon}(v):=\phi(\varepsilon, v)
$$

for $\varepsilon \in J$ and $v \in V$. Then, the system (2.14)-(2.15) in the unknowns $\varepsilon$ and $v$ can be written

$$
P F_{\varepsilon}\left(v+\phi_{\varepsilon}(v)\right)=\delta_{\varepsilon}(v) v, \quad\left\|v+\phi_{\varepsilon}(v)\right\|^{2}=1
$$

while the identities (2.12) and (2.13) become

$$
\begin{gathered}
\left\langle P F_{\varepsilon}\left(v+\phi_{\varepsilon}(v)\right), v\right\rangle=\delta_{\varepsilon}(v)\|v\|^{2}, \\
Q F_{\varepsilon}\left(v+\phi_{\varepsilon}(v)\right)=\delta_{\varepsilon}(v) \phi_{\varepsilon}(v) .
\end{gathered}
$$

We now show, using (BG), that for any $\varepsilon$ there exist (at least) two distinct solutions $v=v_{\varepsilon}, z=z_{\varepsilon}$ of (2.16). For this purpose, let $B=\nabla b$. Then $F_{\varepsilon}=\nabla f_{\varepsilon}$ with

$$
f_{\varepsilon}(x)=\frac{1}{2}\langle A x, x\rangle+\varepsilon b(x) .
$$

Our claim - suggested by [13] - is that for any fixed $\varepsilon \in J$ sufficiently small the solutions $v \in V$ of (2.16) are precisely the critical points of the $C^{1}$ functional $\alpha_{\varepsilon}$ defined by

$$
\alpha_{\varepsilon}(v):=f_{\varepsilon}\left(v+\phi_{\varepsilon}(v)\right)=\frac{1}{2}\left\langle A \phi_{\varepsilon}(v), \phi_{\varepsilon}(v)\right\rangle+\varepsilon b\left(v+\phi_{\varepsilon}(v)\right), \quad v \in V,
$$

over

$$
M_{\varepsilon}=\left\{v \in V:\left\|v+\phi_{\varepsilon}(v)\right\|^{2}=1\right\},
$$

which, for $\varepsilon$ small, is a $C^{1}$ manifold. To see this, for any $\varepsilon \in J$ put

$$
\beta_{\varepsilon}(v)=\left\|v+\phi_{\varepsilon}(v)\right\|^{2}, \quad v \in \bar{V},
$$

and observe that the gradient of the functional $v \mapsto \beta_{0}(v)=\|v\|^{2}$ does not vanish on $\bar{V}$, since this set does not contain the origin. Therefore, because of the compactness of $\bar{V}$, the gradient of $\beta_{\varepsilon}$ remains different from zero on $V$ for $\varepsilon$ sufficiently small. This shows that for these values of $\varepsilon$ the set $M_{\varepsilon}$ is actually a $C^{1}$ manifold. We shall assume that $J$ is such that any $\varepsilon \in J$ has this property. 
At a critical point $v_{0}$ of $\alpha_{\varepsilon}$ on $M_{\varepsilon}$, we have

$$
\alpha_{\varepsilon}^{\prime}\left(v_{0}\right) h=\frac{\mu}{2} \beta_{\varepsilon}^{\prime}\left(v_{0}\right) h
$$

for all $h \in N$ and for some Lagrange multiplier $\mu$. Now compute the first and second member of (2.21) by the chain rule; we have

$$
\begin{aligned}
\alpha_{\varepsilon}^{\prime}\left(v_{0}\right) h & =f_{\varepsilon}^{\prime}\left(v_{0}+\phi_{\varepsilon}\left(v_{0}\right)\left[h+\phi_{\varepsilon}^{\prime}\left(v_{0}\right) h\right]\right. \\
& =\left\langle F_{\varepsilon}\left(v_{0}+\phi_{\varepsilon}\left(v_{0}\right)\right), h+\phi_{\varepsilon}^{\prime}\left(v_{0}\right) h\right\rangle \\
& =\left\langle P F_{\varepsilon}\left(v_{0}+\phi_{\varepsilon}\left(v_{0}\right)\right)+Q F_{\varepsilon}\left(v_{0}+\phi_{\varepsilon}\left(v_{0}\right)\right), h+\phi_{\varepsilon}^{\prime}\left(v_{0}\right) h\right\rangle \\
& =\left\langle P F_{\varepsilon}\left(v_{0}+\phi_{\varepsilon}\left(v_{0}\right)\right), h\right\rangle+\left\langle Q F_{\varepsilon}\left(v_{0}+\phi_{\varepsilon}\left(v_{0}\right), \phi_{\varepsilon}^{\prime}\left(v_{0}\right) h\right\rangle .\right.
\end{aligned}
$$

Using (2.18) it follows that

$$
\alpha_{\varepsilon}^{\prime}\left(v_{0}\right) h=\left\langle P F_{\varepsilon}\left(v_{0}+\phi_{\varepsilon}\left(v_{0}\right)\right), h\right\rangle+\delta_{\varepsilon}\left(v_{0}\right)\left\langle\phi_{\varepsilon}\left(v_{0}\right), \phi_{\varepsilon}^{\prime}\left(v_{0}\right) h\right\rangle .
$$

On the other hand,

$$
\begin{aligned}
\beta_{\varepsilon}^{\prime}\left(v_{0}\right) h & =2\left\langle v_{0}+\phi_{\varepsilon}\left(v_{0}\right), h+\phi_{\varepsilon}^{\prime}\left(v_{0}\right) h\right\rangle \\
& =2\left[\left\langle v_{0}, h\right\rangle+\left\langle\phi_{\varepsilon}\left(v_{0}\right), \phi_{\varepsilon}^{\prime}\left(v_{0}\right) h\right\rangle\right] .
\end{aligned}
$$

Replacing in (2.21) we then have

$$
\begin{gathered}
\left\langle P F_{\varepsilon}\left(v_{0}+\phi_{\varepsilon}\left(v_{0}\right)\right), h\right\rangle+\delta_{\varepsilon}\left(v_{0}\right)\left\langle\phi_{\varepsilon}\left(v_{0}\right), \phi_{\varepsilon}^{\prime}\left(v_{0}\right) h\right\rangle \\
=\mu\left(\left\langle v_{0}, h\right\rangle+\left\langle\phi_{\varepsilon}\left(v_{0}\right), \phi_{\varepsilon}^{\prime}\left(v_{0}\right) h\right\rangle\right), \quad h \in N .
\end{gathered}
$$

Putting $h=v_{0}$ and using (2.17), it follows in particular that

$$
\delta_{\varepsilon}\left(v_{0}\right)\left\|v_{0}\right\|^{2}+\delta_{\varepsilon}\left(v_{0}\right)\left\langle\phi_{\varepsilon}\left(v_{0}\right), \phi_{\varepsilon}^{\prime}\left(v_{0}\right) v_{0}\right\rangle=\mu\left(\left\|v_{0}\right\|^{2}+\left\langle\phi_{\varepsilon}\left(v_{0}\right), \phi_{\varepsilon}^{\prime}\left(v_{0}\right) v_{0}\right\rangle\right) .
$$

This shows that $\mu=\delta_{\varepsilon}\left(v_{0}\right)$, and replacing this in (2.22) yields

$$
\left\langle P F_{\varepsilon}\left(v_{0}+\phi_{\varepsilon}\left(v_{0}\right)\right), h\right\rangle=\delta_{\varepsilon}\left(v_{0}\right)\left\langle v_{0}, h\right\rangle
$$

for all $h \in N$, showing that $v_{0}$ satisfies (2.16), as claimed.

Now observe that for $v \in V, \phi_{\varepsilon}(v)=\phi(\varepsilon, v) \rightarrow \phi(0, v)=0$ as $\varepsilon \rightarrow 0$. The definitions (2.9) and (2.20) then show that $M_{\varepsilon} \subset M$ for $\varepsilon$ sufficiently small. It follows in particular that $M_{\varepsilon}$ is compact; and thus for each $\varepsilon$ there exist $v_{\varepsilon}, z_{\varepsilon} \in M_{\varepsilon}$ such that

$$
\alpha_{\varepsilon}\left(v_{\varepsilon}\right)=\min _{v \in M_{\varepsilon}} \alpha_{\varepsilon}(v), \quad \alpha_{\varepsilon}\left(z_{\varepsilon}\right)=\max _{v \in M_{\varepsilon}} \alpha_{\varepsilon}(v)
$$

Therefore, for each $\varepsilon, v_{\varepsilon}$ and $z_{\varepsilon}$ solve (2.16).

It is now quite easy to conclude that $S_{0}$ has at least one bifurcation point: we can use for this an argument already employed in [3]. Take a sequence $\left\{\varepsilon_{n}\right\}$ with $\varepsilon_{n} \neq 0$ for each $n$ and $\varepsilon_{n} \rightarrow 0$ as $n \rightarrow \infty$. Let $v_{n}:=v_{\varepsilon_{n}}, \delta_{n}:=\delta_{\varepsilon_{n}}\left(v_{n}\right)=\delta\left(\varepsilon_{n}, v_{n}\right)$ and $\phi_{n}:=$ $\phi_{\varepsilon_{n}}\left(v_{n}\right)=w\left(\delta_{n}, \varepsilon_{n}, v_{n}\right)$; then putting $x_{n}:=v_{n}+\phi_{n}$, we have a sequence $\left\{\left(\delta_{n}, \varepsilon_{n}, x_{n}\right)\right\}$ with $x_{n} \in S$ for each $n \in n$ and

$$
A x_{n}+\varepsilon_{n} B\left(x_{n}\right)=\delta_{n} x_{n} \quad \forall n \in n .
$$


By the compactness of $M$, we can assume - passing if necessary to a subsequence that $v_{n} \rightarrow v_{0} \in M \subset V$. Consequently,

$$
\delta_{n} \rightarrow \delta\left(0, v_{0}\right)=0, \quad \phi_{n} \rightarrow w\left(0,0, v_{0}\right)=0 .
$$

It follows that $x_{n} \rightarrow v_{0}$, which in turn implies that $v_{0} \in S$ and - by (2.24) - that $A v_{0}=0$, showing that $v_{0} \in S_{0}$ and is a bifurcation point of (1.1). This completes the proof of Theorem 1.1.

We continue our discussion of (1.1) assuming, in addition to the previous hypotheses, that

(BB) $B$ is bounded in a neighbourhood of $S$.

Then we claim that as $\varepsilon \rightarrow 0$, the family $\left(v_{\varepsilon}\right)$ of minimizers of $\alpha_{\varepsilon}$ on $M_{\varepsilon}-(\operatorname{see}(2.23))-$ converges (through a sequence) to a minimizer $v_{0}$ of $b$ on $S_{0}$, and similarly for $\left(z_{\varepsilon}\right)$. Before stating this formally, we need to list some facts about the functional

$$
h_{\varepsilon}:=\frac{\alpha_{\varepsilon}}{\varepsilon}, \quad \varepsilon \neq 0
$$

(i) Since $v_{\varepsilon}$ minimizes $\alpha_{\varepsilon}$ on $M_{\varepsilon}$, then it also minimizes $h_{\varepsilon}$ for $\varepsilon>0$ : that is

$$
h_{\varepsilon}\left(v_{\varepsilon}\right) \leq h_{\varepsilon}(v), \quad \forall \varepsilon>0, \forall v \in M_{\varepsilon} .
$$

(ii) The assumption (BB) implies that $\phi_{\varepsilon}(v)=O(|\varepsilon|)$ - that is, $\left\|\phi_{\varepsilon}(v)\right\| \leq K|\varepsilon|$ for some $K>0-$ as $\varepsilon \rightarrow 0$, uniformly w.r.t. $v$ in a bounded neighbourhood of $S_{0}$. To see this, recall the definition of $\phi_{\varepsilon}(v)=\phi(\varepsilon, v)$ via $(2.11)$ and recall that $w(\delta, \varepsilon, v)$ solves (2.4), that is $A w-\delta w=-\varepsilon Q B(v+w)$ or, putting $H_{\delta}=-\left(\left.(A-\delta I)\right|_{W}\right)^{-1}$,

$$
w=\varepsilon H_{\delta} Q B(v+w) .
$$

As $H_{\delta}$ and $Q$ are bounded linear operators and $B$ is bounded in a neighbourhood of $S$, it follows that $w(\delta, \varepsilon, v)=O(|\varepsilon|)$ uniformly with respect to $(\delta, v)$ in a bounded neighbourhood of $\{0\} \times S_{0} \subset \mathbb{R} \times N$. This implies in particular the desired bound for $\left\|\phi_{\varepsilon}(v)\right\|$.

(iii) For each $\varepsilon>0$ small and each $y \in S_{0}$, there exists a number $t=t(\varepsilon, y)$, with $0<t \leq 1$, such that $t(\varepsilon, y) y \in M_{\varepsilon}$; moreover, $t(\varepsilon, y) \rightarrow 1$ as $\varepsilon \rightarrow 0$ for each $y \in S_{0}$. Indeed pick an $y \in S_{0}$ and consider the point ty running on the ray from 0 through $y$; by (2.20) we see that it meets $M_{\varepsilon}$ if and only if

$$
t^{2}+\left\|\phi_{\varepsilon}(t y)\right\|^{2}=1 .
$$

The remark made above about $\phi_{\varepsilon}$ implies that this equation has (for each $\varepsilon$ small) a solution in the interval $(0,1]$.

As a consequence of (i) and (iii), we obtain in particular the inequality

$$
h_{\varepsilon}\left(v_{\varepsilon}\right) \leq h_{\varepsilon}(t(\varepsilon, y) y), \quad \varepsilon>0, y \in S_{0} .
$$

(iv) Our next claim is that as $\varepsilon \rightarrow 0, h_{\varepsilon} \rightarrow b$ uniformly in a neighbourhood of $S_{0}$. To see this, consider the expression (2.19) of $\alpha_{\varepsilon}$, which gives

$$
h_{\varepsilon}(v)=\frac{1}{2 \varepsilon}\left\langle A \phi_{\varepsilon}(v), \phi_{\varepsilon}(v)\right\rangle+b\left(v+\phi_{\varepsilon}(v)\right) .
$$


We know from (ii) that $\phi_{\varepsilon}(v)=O(|\varepsilon|)$ as $\varepsilon \rightarrow 0$, uniformly w.r.t. $v$ in a bounded neighbourhood of $S_{0}$. Since $A$ is a bounded linear operator, we have $|\langle A x, x\rangle| \leq C\|x\|^{2}$ for some $C>0$ and all $x \in H$, and therefore $\left\langle A \phi_{\varepsilon}(v), \phi_{\varepsilon}(v)\right\rangle=O\left(\varepsilon^{2}\right)$ as $\varepsilon \rightarrow 0$, uniformly w.r.t. $v$ in a bounded neighbourhood of $S_{0}$. Looking at (2.26), this proves our claim.

We can now establish formally the desired property of $\left(v_{\varepsilon}\right)$.

Proposition 2.1. Let $v_{\varepsilon} \in M_{\varepsilon}$ be such that $\alpha_{\varepsilon}\left(v_{\varepsilon}\right)=\min _{v \in M_{\varepsilon}} \alpha_{\varepsilon}(v)$. Then, there exists a sequence $\left\{v_{n}\right\}:=\left\{v_{\varepsilon_{n}}\right\}$ such that $v_{n} \rightarrow v_{0}$ as $n \rightarrow \infty$, where $v_{0} \in S_{0}$ and satisfies

$$
b\left(v_{0}\right)=\min _{y \in S_{0}} b(y)
$$

Proof. Take any sequence $\left\{\varepsilon_{n}\right\}$ with $\varepsilon_{n}>0$ for each $n$ and $\varepsilon_{n} \rightarrow 0$ as $n \rightarrow \infty$. Let $v_{n}:=v_{\varepsilon_{n}}$ and $h_{n}:=h_{\varepsilon_{n}}$; then we have from (2.25)

$$
h_{n}\left(v_{n}\right) \leq h_{n}\left(t\left(\varepsilon_{n}, y\right) y\right), \quad \forall n \in n, \forall y \in S_{0} .
$$

As before we can assume - passing if necessary to a subsequence - that $v_{n} \rightarrow v_{0} \in S_{0}$. Moreover if $D$ is a neighbourhood of $S_{0}$,

$$
\begin{aligned}
& \left|h_{n}\left(v_{n}\right)-b\left(v_{0}\right)\right| \leq\left|h_{n}\left(v_{n}\right)-b\left(v_{n}\right)\right|+\left|b\left(v_{n}\right)-b\left(v_{0}\right)\right| \leq \sup _{v \in D}\left|h_{n}(v)-b(v)\right| \\
& \quad+\left|b\left(v_{n}\right)-b\left(v_{0}\right)\right|
\end{aligned}
$$

and since - by (iv) above $-h_{n} \rightarrow b$ uniformly in a neighbourhood of $S_{0}$, it follows that $h_{n}\left(v_{n}\right) \rightarrow b\left(v_{0}\right)$ as $n \rightarrow \infty$.

The same argument shows that the right-hand side of (2.27) converges to $b(y)$ as $n \rightarrow \infty$, for each fixed $y \in S_{0}$, because $t\left(\varepsilon_{n}, y\right) \rightarrow 1$ for each $y \in S_{0}$ as proven in (iii). Therefore, letting $n \rightarrow \infty$ in (2.27), we obtain in the limit

$$
b\left(v_{0}\right) \leq b(y), \quad \forall y \in S_{0},
$$

which is the assertion.

Proposition 2.1 leads (as announced in Section 1) to the following improvement of Theorem 1.1 .

THEOREM 2.1. Let T, $B$ and $\lambda_{0}$ be as in Theorem 1.1, and assume, in addition, that $B$ satisfies the assumption (BB). Then, (1.1) admits two bifurcation points from $Z_{0}, v_{0}$ and $v_{1}$, such that

$$
b\left(v_{0}\right)=\min _{y \in S_{0}} b(y), \quad b\left(v_{1}\right)=\max _{y \in S_{0}} b(y) .
$$

Consequently, if b is not constant on $S_{0}$, there exist at least two distinct bifurcation points from $Z_{0}$ for (1.1).

Now a natural question is: can we drop the assumption ' $b$ is not constant on $S_{0}$ ' in the statement of Theorem 2.1? Unable until to give an answer, we present a simple example in which $b$ is constant on $S_{0}$, and still there are infinitely many bifurcation points. 
ExAmple 2.2. Consider (2.1) with $A, B: \mathbb{R}^{3} \rightarrow \mathbb{R}^{3}$ defined as follows. Write $x=$ $\left(x_{1}, x_{2}, z\right)$ for $x \in \mathbb{R}^{3}$ and put

$$
A\left(x_{1}, x_{2}, z\right)=(0,0, z)
$$

In the notations used before, we thus have $v=\left(x_{1}, x_{2}, 0\right)-$ identified with $\left(x_{1}, x_{2}\right) \in$ $\mathbb{R}^{2}-$ and $w=(0,0, z)$ - identified with $z \in \mathbb{R}$. As for $B$, put

$$
B\left(x_{1}, x_{2}, z\right)=\left(2 x_{1} z, 2 x_{2} z, x_{1}^{2}+x_{2}^{2}\right)
$$

and observe that $B=\nabla b$ with $b\left(x_{1}, x_{2}, z\right)=\left(x_{1}^{2}+x_{2}^{2}\right) z$. Equations (2.3) and (2.4) are here respectively

$$
2 \varepsilon z\left(x_{1}, x_{2}\right)=\delta\left(x_{1}, x_{2}\right)
$$

and

$$
z+\varepsilon\left(x_{1}^{2}+x_{2}^{2}\right)=\delta z
$$

Solve the latter uniquely with respect to $z$ :

$$
z=\frac{\varepsilon}{\delta-1}\left(x_{1}^{2}+x_{2}^{2}\right)
$$

so that - putting $x_{1}^{2}+x_{2}^{2}=R^{2}-$ we have

$$
z=w(\delta, \varepsilon, v)=w(\delta, \varepsilon,\|v\|)=\frac{\varepsilon}{\delta-1} R^{2}:=w(\delta, \varepsilon, R) .
$$

Using this and (2.28) yield the equation in $\delta$ :

$$
\delta=2 \varepsilon z=\frac{2 \varepsilon^{2}}{\delta-1} R^{2}
$$

that can be written

$$
\delta^{2}-\delta-2 \varepsilon^{2} R^{2}=0
$$

and can thus be explicitly solved to give

$$
\delta=\delta(\varepsilon, R)=\frac{1}{2}\left(1-\sqrt{1+8 \varepsilon^{2} R^{2}}\right) .
$$

Replacing this in (2.29) permits us to compute the function $\phi$ appearing in the formulae (2.16)-(2.18). Here we have $\phi(\varepsilon, v)=\phi(\varepsilon,\|v\|)=w(\delta(\varepsilon, R), \varepsilon, R):=\phi(\varepsilon, R)$ with

$$
\phi(\varepsilon, R)=\frac{\varepsilon R^{2}}{\frac{1}{2}\left(1-\sqrt{1+8 \varepsilon^{2} R^{2}}\right)-1}=-\frac{2 \varepsilon R^{2}}{1+\sqrt{1+8 \varepsilon^{2} R^{2}}} .
$$

In turn, this allows us to determine $M_{\varepsilon}$ by (2.20), which becomes here

$$
R^{2}+\frac{4 \varepsilon^{2} R^{4}}{\left(1+\sqrt{1+8 \varepsilon^{2} R^{2}}\right)^{2}}=1
$$


This equation has for each $\varepsilon \neq 0$ one solution $R(\varepsilon)$ with $0<R(\varepsilon)<1$, and moreover $1-R(\varepsilon)^{2}=O\left(\varepsilon^{2}\right)$ as $\varepsilon \rightarrow 0$. We see in particular that $M_{\varepsilon}$ is a circle in the plane $\left(x_{1}, x_{2}\right)$ approaching $S_{0}$ (the circle of radius 1 ) as $\varepsilon \rightarrow 0$. Replacing this value $R(\varepsilon)$ in the above expressions (2.30) and (2.31) produces the functions

$$
\delta(\varepsilon):=\delta(\varepsilon, R(\varepsilon)), \quad \phi(\varepsilon):=\phi(\varepsilon, R(\varepsilon)),
$$

which finally allow us to express the solutions $(\delta, \varepsilon, x)$ in terms of the sole parameter $\varepsilon$. Indeed, these are (for any $\varepsilon$ )

$$
(\delta(\varepsilon), \varepsilon, x(\varepsilon)) \quad \text { with } \quad x(\varepsilon)=(v, z), \quad\|v\|=R(\varepsilon), \quad z=\phi(\varepsilon) .
$$

It follows in particular that each $v_{0} \in S_{0}$ is a bifurcation point: just take

$$
x(\varepsilon)=\left(R(\varepsilon) v_{0}, \phi(\varepsilon)\right)
$$

to have a family of nontrivial solutions that converges to $\left(v_{0}, 0\right)$ as $\varepsilon \rightarrow 0$.

\section{REFERENCES}

1. M. S. Berger, Nonlinearity and functional analysis (Academic Press, New York, 1977).

2. R. Chiappinelli, M. Furi and M. P. Pera, Normalized eigenvectors of a perturbed linear operator via general bifurcation, Glasgow Math. J. 50 (2008), 303-318.

3. R. Chiappinelli, M. Furi and M. P. Pera, Topological persistence of the normalized eigenvectors of a perturbed self-adjoint operator, Appl. Math. Lett. 23 (2010), 193-197.

4. R. Chiappinelli, M. Furi and M. P. Pera, A new theme in nonlinear analysis: Continuation and bifurcation of the unit eigenvectors of a perturbed linear operator, Commun. Appl. Anal. 15(2-4) (2011), 299-312.

5. M. G. Crandall and P. H. Rabinowitz, Bifurcation from simple eigenvalues, J. Funct. Anal. 8 (1971), 321-340.

6. K. Deimling, Nonlinear functional analysis (Springer, New York, 1985).

7. M. Furi, M. Martelli and M. P. Pera, General bifurcation theory: Some local results and applications, in Differential equations and applications to biology and to industry (Cooke, $\mathrm{K}$., Cumberbatch, E., Martelli, M, Tang, B. and Thieme, H., Editors) (World Scientific, River Edge, NJ, 1996), 101-115.

8. M. Furi and M. P. Pera, Cobifurcating branches of solutions for nonlinear eigenvalue problems in Banach spaces, Ann. Mat. Pura Appl. 135(4) (1983), 119-131.

9. A. Granas and J. Dugundji, Fixed point theory, Springer Monographs in Mathematics (Springer, New York, 2003).

10. M. A. Krasnoselskii, Topological methods in the theory of nonlinear integral equations (Macmillan, New York, 1964).

11. G. Prodi and A. Ambrosetti, Analisi non lineare, Scuola Normale Superiore Pisa, Quaderno 1 (1973).

12. P. H. Rabinowitz, Minimax methods in critical point theory with applications to differential equations, CBMS Regional Conference Series Math., vol. 65 (AMS, Providence, RI, 1986).

13. C. A. Stuart, An introduction to bifurcation theory based on differential calculus, in Nonlinear analysis and mechanics: Heriot-Watt Symposium, Vol. 4 (Res. Notes Math. vol. 39; Pitman, London, 1979), 76-135. 